\title{
Iron-Restricted Erythropoiesis in a Population of Elderly Hospitalized Anemic Patients
}

\section{Torbjörn Karlsson}

Department of Hematology, Uppsala University Hospital, Uppsala, Sweden.

Email: torbjorn.a.karlsson@akademiska.se

Received April 19 $9^{\text {th }}, 2012$; revised May 15 $5^{\text {th }}, 2012$; accepted May 26 ${ }^{\text {th }}, 2012$

\begin{abstract}
The aim of this observational study was to assess the prevalence of iron-restricted erythropoiesis (IRE) in a population of elderly hospitalized anemic patients. Prevalence of IRE was found to be $41 \%$ and the most common clinical diagnoses in such patients were hematologic malignancies and solid tumors. Comparison between patients with IRE and non-IRE patients revealed that mean corpuscular volume, mean corpuscular hemoglobin, iron, and transferrin saturation were significantly lower in the IRE group, whereas no significant difference was found for $\mathrm{Hb}$, transferrin, or ferritin. There was a more pronounced inflammatory response in the IRE group demonstrated by a higher C-reactive protein level.
\end{abstract}

Keywords: Anemia; Iron-Restriction; Elderly Patients

\section{Introduction}

Several different mechanisms can restrict erythropoiesis by supply of iron to the erythroblasts [1]. In absolute iron deficiency anemia, erythropoiesis is restricted by the depleted iron stores, whereas an inflammatory response induces iron-restricted erythropoiesis (IRE) by iron-sequestration [1], commonly defined as ferritin $>100 \mu \mathrm{g} / \mathrm{L}$ plus transferrin saturation (TSAT) $\leq 20 \%$ [2] or a bone marrow sample staining positive for iron in combination with TSAT $\leq 20 \%$. The molecular mechanism by which an inflammatory response associated with autoimmune diseases, cancer, or infections induces blockage of intestinal iron absorption and efflux from reticuloendothelial system (RES) macrophages has been elucidated in recent years $[3,4]$. An inflammatory response increases expression of the hepatic protein hepcidin in an interleukin-6dependent manner [5]. Secreted hepcidin binds to the transmembrane iron transporter ferroportin in macrophages of the RES as well as in hepatocytes and enterocytes, down-regulating its expression by post-translational mechanisms [6]. In addition to decreased transport of iron from enterocytes to the circulation, the downregulated ferroportin reduces iron efflux from macrophages $[7,8]$, thus making less iron available for erythropoiesis, the ultimate consequence of which is IRE and anemia [9]. Most of our current knowledge of IRE and its treatment with iron supplementation stems from the nephrology setting [10]. In nephrology, IRE is commonly known as functional iron deficiency (FID) and seen in patients with chronic kidney disease (CKD) who are treated with erythropoiesis stimulating agents (ESAs). In these patients, an ESA-driven supraphysiological erythropoiesis is restricted by the delivery of stored iron to the bone marrow erythroblasts, which can lead to ESA-hyporesponsiveness. This condition can be successfully treated by intravenous (iv) iron supplementation [10]. FID is also seen in cancer patients on ESA therapy, and the addition of iv iron has been found to augment the erythropoietic response in this patient group [11].

\section{Material and Methods}

This study was conducted at the Department of Medicine, Capio Sankt Görans Hospital, Stockholm, Sweden. One hundred and four newly diagnosed anemic patients $(\mathrm{Hb}<$ $134 \mathrm{~g} / \mathrm{L}$ in men and $<117 \mathrm{~g} / \mathrm{L}$ in women) 60 years of age or older admitted to the hospital were screened for IRE, defined as a bone marrow smear staining positive for iron in combination with TSAT $\leq 20 \%$. This observational study was performed on blood and bone marrow samples collected during routine anemia investigation and all procedures were in accordance with the Helsinki Declaration of 1964. Patients receiving iron supplementation or red cell transfusions were excluded from the study. Bone marrow smears were stained by means of the May-Grunwald-Giemsa method, and bone marrow iron stores were investigated using Prussian blue staining. Patients with no stainable bone marrow iron $(\mathrm{n}=38)$ were diagnosed as having absolute iron deficiency. The diagnosis for 
those with stainable iron was anemia of chronic disease (ACD) or other non-iron deficiency anemia. The iron replete patients were divided into two groups: those with IRE $(\mathrm{n}=43)$ and those with non-IRE anemia (bone marrow staining positive for iron + TSAT $>20 \%)(n=23)$. Hematological data, biochemical iron status and C-reactive protein (CRP) were compared between the IRE and non-IRE groups. Complete blood counts were performed using the ADVIA 2120 analyzer (Siemens Diagnostics, Deerfield, IL, USA). CRP, iron, and transferrin were investigated by means of ADVIA 2400 and sTfR using BN ProSpec (Siemens Diagnostics, Deerfield, IL, USA). Ferritin was analyzed using ADVIA Centaur XP. All reagents were from Siemens Diagnostics, with the exception of the transferrin assay, which was produced by Dakopatts (Dakopatts, Dorchester, UK). Laboratory reference values for $\mathrm{Hb}$ is $>134 \mathrm{~g} / \mathrm{L}$ for men and $>117 \mathrm{~g} / \mathrm{L}$ for women, mean corpuscular volume (MCV) 82 - $98 \mathrm{fL}$, mean corpuscular hemoglobin $(\mathrm{MCH}) 27$ - 33 pg, iron 9 $34 \mu \mathrm{mol} / \mathrm{L}$, transferrin $1.9-3.2 \mathrm{~g} / \mathrm{L}$, TSAT $15 \%-60 \%$ for men and $10 \%-50 \%$ for women, ferritin $20-375$ $\mu \mathrm{g} / \mathrm{L}$ for men and $7-120 \mu \mathrm{g} / \mathrm{L}$ for women, CRP $<5$ $\mathrm{mg} / \mathrm{L}$ and soluble transferrin receptor (sTfR) $0.8-1.7$ $\mathrm{mg} / \mathrm{L}$. All analyses were performed at the Department of Clinical Chemistry, Capio Sankt Görans Hospital, with the exception of the bone marrow analyses, which were performed at the Department of Pathology, Capio Sankt Görans Hospital. Statistical analyses were conducted using the SigmaPlot 11 software package (Systat Software, San Jose, CA, USA). Quantitative variables were expressed as means +/- standard deviations. The Student's t test or Mann-Whitney rank sum test was used to compare the variables between the two groups. A $p$ value of less than 0.05 was considered statistically significant.

\section{Results and Discussion}

The aim of this observational study was to assess the prevalence of IRE in a population $(n=104)$ of elderly hospitalized anemic patients and to compare hematological and biochemical iron data between the IRE and non-IRE groups. The prevalence of IRE in this population was $41 \%(43 / 104)$. The mean age in the IRE group was 83 years versus 78 in the non-IRE group. This age difference was not statistically significant. The main clinical diagnoses among the anemic patients with IRE were hematologic malignancies $(26 \%)$, solid tumors (18\%), and infections (17\%) (Table 1), while in the nonIRE group, infections $(22 \%)$, CKD $(22 \%)$ and liver disease $(17 \%)$ were the most common (Table 1). Interestingly, 44\% (20/43) of the patients in the IRE group suffered from hematologic malignancies or solid tumors compared to $13 \%(3 / 23)$ in the non-IRE group. The explanation behind this difference is unclear, but it is possible that malignant cells induce a cytokine "profile" in
Table 1. Clinical diagnoses of patients with IRE anemia (positive bone marrow iron staining + TSAT $\leq 20 \%$ ) versus non-IRE anemia (positive bone marrow iron staining + TSAT > 20\%). Abbreviations: IRE, iron-restricted erythropoiesis; TSAT, transferrin saturation; CKD, chronic kidney disease; FOU, fever of unknown origin; GCA, giant cell arteritis.

\begin{tabular}{lll}
\hline Clinical diagnosis & IRE & Non-IRE \\
\hline Infection & $5(17 \%)$ & $5(22 \%)$ \\
CKD & $1(2 \%)$ & $5(22 \%)$ \\
Liver disease & $2(4 \%)$ & $4(17 \%)$ \\
Hematologic malignancy & $12(26 \%)$ & $3(13 \%)$ \\
Solid tumor & $8(18 \%)$ & 0 \\
FOU & $6(13 \%)$ & $3(13 \%)$ \\
GCA & $3(7 \%)$ & 0 \\
Miscellanous & $6(13 \%)$ & $3(13 \%)$ \\
\hline
\end{tabular}

the organism that differs from that induced by an inflammatory response associated with non-malignant disease and that these different "profiles" have divergent effects on the iron metabolism [12]. Iron, TSAT, MCV, and $\mathrm{MCH}$ were all significantly lower in the IRE group compared to the non-IRE group (Table 2). The significantly lower iron, TSAT, MCV, and MCH values in the IRE group compared to the non-IRE group indicate a higher degree of iron blockage in the first group (Table 2). No significant differences in $\mathrm{Hb}$, transferrin or ferritin were detected when comparing the two groups (Table 2). The lower TSAT in the IRE group was solely an effect of a lower iron concentration (Table 2). Transferrin is commonly regarded as a "negative" acute-phase reactant [13], but in the population described here, there was no difference between mean transferrin concentrations when comparing the IRE and non-IRE groups, despite a more pronounced inflammatory response in the IRE group determined by a higher mean concentration of CRP (Table 2). The mean concentration of sTfR was significantly higher in the IRE group compared to the non-IRE group (Table 2). The most obvious explanation for this finding is that due to the higher degree of iron blockage in the IRE group, a subsequent lower serum iron concentration stimulates bone marrow erythroblasts to increase their expression of TfR. In previous studies on sTfR levels in ACD, subjects with IRE and non-IRE were not analyzed separately $[14,15]$. The significantly lower $\mathrm{MCH}$ in the IRE group compared to the non-IRE group probably reflects a higher degree of iron restriction in the former. Since $\mathrm{MCH}$ reflects mean red cell hemoglobinization over the life of the red cell, i.e. 120 days, the lower $\mathrm{MCH}$ in the IRE group indicate that the iron restriction is not a phenomenon with an acute onset. Anemia of chronic disease is a common phenomenon associated with conditions such as autoimmune diseases, cancer, and infections [16], and the pathogenetic mechanisms behind IRE 
Table 2. Comparison of hematological and biochemical iron parameters and CRP between groups with IRE (positive bone marrow iron staining + TSAT $\leq 20 \%$ ) and non-IRE anemia (positive bone marrow iron staining + TSAT $>20 \%$ ). Data are expressed as mean $+/-$ SD. Abbreviations: IRE, iron-restricted erythropoiesis; TSAT, transferrin saturation; MCV, mean corpuscular volume; MCH, mean corpuscular hemoglobin; sTfR, soluble transferrin receptor; CRP, C-reactive protein.

\begin{tabular}{llll}
\hline Variable & IRE & Non-IRE & $\boldsymbol{p}$ \\
\hline TSAT $(\%)$ & $10(5)$ & $43(20)$ & $<0.001$ \\
Iron $(\mu \mathrm{mol} / \mathrm{L})$ & $5.2(3.3)$ & $18.2(8.6)$ & $<0.001$ \\
Transferrin $(\mathrm{g} / \mathrm{L})$ & $1.95(0.60)$ & $1.85(0.63)$ & N.S \\
$\mathrm{Hb}(\mathrm{g} / \mathrm{L})$ & $98(14)$ & $99(13)$ & N.S \\
$\mathrm{MCV}(\mathrm{fL})$ & $88.6(7.4)$ & $98.2(8.3)$ & $<0.001$ \\
$\mathrm{MCH}(\mathrm{pg})$ & $28.7(2.9)$ & $32.1(2.6)$ & $<0.001$ \\
Ferritin $(\mu \mathrm{g} / \mathrm{L})$ & $390(387)$ & $548(448)$ & N.S \\
$\mathrm{sTfR}(\mathrm{mg} / \mathrm{L})$ & $1.62(0.66)$ & $1.24(0.67)$ & $<0.01$ \\
$\mathrm{CRP}(\mathrm{mg} / \mathrm{L})$ & $75(65)$ & $35(49)$ & 0.01 \\
\hline
\end{tabular}

partly overlap those in ACD [1,17]. There are limited data on IRE in populations of elderly patients with co-morbid conditions. In a population of anemic oncology patients with a mean age of over 60 years, the prevalence of IRE, determined by ferritin $>100 \mu \mathrm{g} / \mathrm{mL}$ and TSAT $<20 \%$ was $46 \%$ prior to initiation of therapy [18]. One trial reported an IRE prevalence of $27 \%$ in a population of elderly patients undergoing orthopaedic surgery [19]. An IRE prevalence of $35 \%$ was observed in a population of intensive care patients $(n=51)$ [20] that did not only include elderly patients (median age approx. 60). Furthermore, analysis of the percentage of hypochromic erythrocytes has been used to identify subjects (median age 71 years, range 18 - 84 years) with IRE and hyporesponsiveness to ESA in a population of patients with lymphoproliferative malignancies [21]. In the nephrology setting, Mirescu and co-workers [22] demonstrated that in a population of anemic CKD patients (mean age 52 years) without absolute iron deficiency, treatment with iv iron increased $\mathrm{Hb}$ in parallel with an increase in the percentage of patients with TSAT $>20 \%$ from 59 (baseline) to $97 \%$ at the end of the study period. The FAIR-HF trial revealed that intravenous iron significantly improved symptoms and functional capacity in a population of iron deficient patients (mean age approx. 67 years) with congestive heart failure [23]. Subjects in the FAIR-HF trial were considered iron deficient if serum ferritin was (a) less than $100 \mu \mathrm{g} / \mathrm{L}$ or (b) between 100 and $299 \mu \mathrm{g} / \mathrm{L}$ in combination with a transferrin saturation less than $20 \%$. The criterion in (b) suggests that at least a subgroup of patients suffered from IRE but were not reported separately [23]. Intravenous iron therapy has also been found to significantly increase $\mathrm{Hb}$ in anemic patients with rheumatoid arthritis [24].

\section{Conclusion}

The results presented here indicate a $41 \%$ prevalence of IRE in a population of elderly hospitalized anemic patients and that the most common clinical diagnoses associated with IRE are hematologic malignancies and solid tumors. Iron restriction was of the sequestration type, since all patients were iron replete and serum iron was significantly lower in the IRE group. Furthermore, there was a more pronounced inflammatory response in the IRE group determined by the higher level of CRP. These findings and the results of iv iron therapy in IRE outside the hematology/oncology setting highlight the need for clinical trials with iv iron in cancer patients with IRE.

\section{Acknowledgements}

The author is the principal investigator in the FER-FIDCHEMO study (ClinicalTrials.gov.Identifier: NCT0110 1399).

\section{REFERENCES}

[1] L. Goodnough, E. Nemeth and T. Ganz, "Detection, Evaluation, and Management of Iron-Restricted Erythropoiesis," Blood, Vol. 116, No. 23, 2010, pp. 4754-4761. doi:10.1182/blood-2010-05-286260

[2] D. Henry, "Supplemental Iron: A Key to Optimizing the Response of Cancer-Related Anemia to rHuEPO?" The Oncologist, Vol. 3, No. 4, 1998, pp. 275-278.

[3] C. Roy and N. Andrews, "Anemia of Inflammation: The Hepcidin Link," Current Opinion in Hematology, Vol. 12, No. 2, 2005, pp. 107-111. doi:10.1097/00062752-200503000-00001

[4] E. Nemeth and T. Ganz, "Hepcidin and Iron-Loading Anemias," Haematologica, Vol. 91, No. 6, 2006, pp. 727732.

[5] E. Nemeth, S. Rivera, V. Gabayan, C. Keller, S. Taudorf, K. Pedersen and T. Ganz, "IL-6 Mediates Hypoferremia of Inflammation by Inducing the Synthesis of the Iron Regulatory Hormone Hepcidin," Journal of Clinical Investigation, Vol. 113, No. 9, 2004, pp. 1271-1276.

[6] E. Nemeth, M. Tuttle, J. Powelson, M. Vaughn, A. Donovan, D. McVey Ward, T. Ganz and J. Kaplan, "Hepcidin Regulates Cellular Iron Efflux by Binding to Ferroportin and Inducing Its Internalization," Science, Vol. 306, No. 5704, 2004, pp. 2090-2093. doi:10.1126/science. 1104742

[7] S. Rivera, L. Liu, E. Nemeth, V. Gabayan, O. Sorensen and T. Ganz, "Hepcidin Excess Induces the Sequestration of Iron and Exacerbates Tumor-Associated Anemia," Blood, Vol. 105, No. 4, 2005, pp. 1797-1802. doi:10.1182/blood-2004-08-3375

[8] L. Viatte, G. Nicolas, D-Q. Lou, M. Bennoun, J.-C. Lebordes Brion, F. Cannone Hergaux, K. Schönig, H. Bujard, A. Kahn, N. Andrews and S. Vaulont, "Chronic Hepcidin Induction Causes Hypoferremia and Alters the Pattern of Cellular Iron Accumulation in Hemochroma- 
totic Mice," Blood, Vol. 107, No. 7, 2006, pp. 2952-2958. doi:10.1182/blood-2005-10-4071

[9] D. Weinstein, C. Roy, M. Fleming, M. Loda, J. Wolfsdorf and N. Andrews, "Inappropriate Expression of Hepcidin Is Associated with Iron Refractory Anemia: Implications for the Anemia of Chronic Disease," Blood, Vol. 100, No. 10, 2002, pp. 3776-3781. doi:10.1182/blood-2002-04-1260

[10] F. Locatelli, A. Covic, K-U. Eckardt, A. Wiecek, R. Wanholder and on Behalf of the ERA-EDTA Advisory Board, "Anemia Management in Patients with Chronic Kidney Disease: A Position Statement by the Anemia Working Group of European Renal Best Practice," $\mathrm{Ne}$ phrology Dialysis Transplantation, Vol. 24, No. 2, 2009, pp. 348-354. doi:10.1093/ndt/gfn653

[11] T. Karlsson, "Effects of Iron Supplementation on Erythropoietic Response in Patients with Cancer-Associated Anemia Treated by Means of Erythropoietic Stimulating Agents," International Scholarly Research Network Hematology, Vol. 2011, No. 2011, 2011, Article ID 108397 , 6 p. http://hindawi.com/journals/ane/2011/925907

[12] G. Weiss, "Iron and Immunity: A Double Egged Sword," European Journal of Clinical Investigation, Vol. 32, Suppl. 1, 2002, pp. 70-78. doi:10.1046/j.1365-2362.2002.0320s1070.x

[13] A. Erslev, "Anemia of Chronic Disease," In: E. Beutler, M. Lichtmann, B. Coller, T. Kipps and U. Seligsohn, Eds., Williams Hematology, 6th Edition, McGraw-Hill, New York, 2001, pp. 481-487.

[14] K. Punnonen, K. Irjala and A. Rajamäki, "Iron-Deficiency Anemia Is Associated with High Concentrations of Transferrin Receptor in Serum," Clinical Chemistry, Vol. 40, No. 5, 1994, pp. 774-776.

[15] K. Punnonen, K. Irjala andA. Rajamäki, "Serum Transferrin Receptor and Its Ratio to Serum Ferritin in the Diagnosis of Iron Deficiency," Blood, Vol. 89, No. 3, 1997, pp. 1052-1057.

[16] G. Weiss and L. Goodnough, "Anemia of Chronic Disease," New England Journal of Medicine, Vol. 352, No. 10, 2005, pp. 1011-1023. doi:10.1056/NEJMra041809

[17] C. Thomas and L. Thomas, "Anemia of Chronic Disease: Pathophysiology and Laboratory Diagnosis," Laboratory Hematology, Vol. 11, No. 1, 2005, pp. 14-23.
doi:10.1532/LH96.04049

[18] D. Henry, N. Dahl and on Behalf of the Ferrleict Cancer Study Group, "Iron or Vitamin $\mathrm{B}_{12}$ Deficiency in Anemic Patients Prior to Erythropoiesis-Stimulating Agent Therapy," Community Oncology, Vol. 4, No. 2, 2007, pp. 95-101. doi:10.1016/S1548-5315(11)70057-2

[19] M. Basora, R. Delofeu, F. Salazar, L. Quinto and C. Gomar, "Improved Preoperative Iron Status Assessment by Soluble Transferrin Receptor in Elderly Patients Undergoing Knee and Hip Replacement," Clinical and LaboratoryHaematology, Vol. 28, No. 6, 2006, pp. 370-375. doi:10.1111/j.1365-2257.2006.00821.x

[20] M. Patteril, A. Davey-Quinn, J. Gedney, S. Murdoch andM. Bellamy, "Functional Iron Deficiency, Infection and Systemic Inflammatory Response Syndrome in Criticall Illness," Anaesthia and Intensive Care, Vol. 29, No. 5, 2001, pp. 473-479.

[21] E. Katodritou, E. Terpos, K. Zervas, M. Speletas, D. Kapetanos, C. Kartsios, E. Verrou, A. Banti, S. Effraimidou and J. Christakis, "Hypochromic Erythrocytes (\%): A Reliable Marker for Regognizing Iron-Restricted Erythropoiesis and Predicting Response to Erythropoietin in Anemic Patients with Myeloma and Lymphoma," Annals of Hematology, Vol. 86, No. 5, 2007, pp. 369-376. doi:10.1007/s00277-007-0258-X

[22] G. Mirescu, L. Garneata, C. Capusa and N. Ursea, "Intravenous Iron Supplementation for the Treatment of Anaemia in Pre-Dialyzed Chronic Renal Failure Patients," Nephrology Dialysis Transplantation, Vol. 21, No. 1, 2005, pp. 120-124. doi:10.1093/ndt/gfi087

[23] S. Anker, J. Comin Colet, G. Fillipatos, R. Willenheimer, K. Dickstein, R. Drexler, T. Luscher, B. Bart, W. Banasiak, J. Niegowska, B-A. Kirwan, C. Mori, B. von Eisenhart Rothe, S. Pocock, P. Poole-Wilson and P. Ponikowski, "Ferric Carboxymaltose in Patients with Heart Failure and Iron Deficiency," New England Journal of Medicine, Vol. 361, No. 25, 2009, pp. 2436-2447. doi:10.1056/NEJMoa0908355

[24] D. Bentley and P. Williams, "Parenteral Iron Therapy in the Anaemia of Rheumatoid Arthritis," Rheumatology and Rehabilitation, Vol. 21, No. 2, 1982, pp. 88-92. doi:10.1093/rheumatology/21.2.88 